\title{
Role of p16, Ki67 and CK17 in differentiating benign lesions, cervical intraepithelial neoplasia(CIN) and atypical immature squamous metaplasia(AIM) of uterine cervix
}

\author{
Mitra S.K. ${ }^{1}$, Misra R.K. ${ }^{2}$, Varshney B. ${ }^{3}$, Kumari A. ${ }^{4}$, Rai D. ${ }^{5}$ \\ ${ }^{1}$ Dr. Shaila K Mitra, MD, Associate Professor, Department of Pathology, ${ }^{2}$ Dr. Rajiv K Misra, Professor and Head, \\ Department of Pathology, ${ }^{3}$ Dr. Bharti Varshney, Resident, Department of Pathology, ${ }^{4}$ Dr. Ankita Kumari, Lecturer, \\ Department of Obstretics and Gynaecology, ${ }^{5}$ Dr. Divya Rai, Resident, Department of Pathology, all authors are affiliated \\ with B.R.D. Medical College, Gorakhpur, UP, India
}

Address for Correspondence: Dr. Shaila Kumari Mitra, Associate Professor, Department of Pathology, B.R.D. Medical College, Gorakhpur. UP, Email: shaila.prasad14@yahoo.in

\begin{abstract}
Cervical intraepithelial neoplasia (CIN) is a premalignant lesion capable of progressing to cervical cancer. Despite the existing well-defined criteria, the histopathological diagnosis is subject to high rates of discordance among pathologists. Aim: To study the role of p16, Ki67 and CK17 in differentiating benign lesions, cervical intraepithelial lesions(CIN) and atypical immature squamous metaplasia (AIM)and to improve intra and interobserver reproducibility of diagnosis of cervical neoplasia. Material and Methods: In a cross sectional study, a total of 75 cervical biopsies including benign lesions ( $n=24)$, AIM ( $n=28), \operatorname{CIN}(n=23)$ were studied and analyzed immunohistochemically using p16, Ki67 and CK17 immunomarkers. Data was evaluated using chi-square test. Results: p16 and Ki 67positivity were observed in $91.3 \%$ and $78.26 \%$ of CIN and $28.57 \%$ of AIM respectively. None of the benign lesions expressed p16 and Ki67while CK17 positivity was observed in $46.42 \%$ of CIN and $100 \%$ of AIM with $12.5 \%$ of benign lesions. Conclusion: The three biomarkers (p16, CK17 and Ki67) had a high degree of sensitivity and specificity and appear to be a useful and reliable diagnostic adjunct to improve the routine diagnosis and reduce interobserver variability in cervical biopsy specimens. Immunohistochemical markers such as p16 alone or with Ki67 represents important tool for the pathologists in distinguishing high grade cervical dysplasia from its benign mimics such as AIM and reactive inflammatory lesion thus avoiding overtreatment.
\end{abstract}

Key words: p16 ${ }^{\text {ink4a }}$, Cytokeratin17, Ki67, Cervical intraepithelial neoplasia, Cervix uteri

\section{Introduction}

Cervical cancer is the commonest cancer cause of death among women in developing countries [1]. Although introduction of pap in developed countries has been effective in reducing cervical cancer mortality and morbidity rates, the efficacy of pap test is hampered by high interobserver variability and high false negative and false positive rate, the range between $20-30 \%$ and $50-70 \%$ respectively [2,3]. Cervical cancer is caused by Human Papilloma virus (HPV), infection of which is acquired by about $80 \%$ of sexually active women by 50 years of age [4]. Almost all of the invasive cervical cancers are preceded by cervical intraepithelial

Manuscript received: $18^{\text {th }}$ December 2016

Reviewed: $26^{\text {th }}$ December 2016

Author Corrected: $05^{\text {th }}$ January 2017

Accepted for Publication: $12^{\text {th }}$ January 2017 neoplasia (CIN) [5,6]. Persistent infections with high risk human papilloma virus (hr-HPV) types lead to CIN and invasive cancer [7]. Despite well-defined criteria, the histopathologic diagnosis is subject to high rates of discrepancy among pathologists [8-10]. Supplementary methods using objective biomarkers are needed to achieve more accurate diagnosis.

The term atypical immature sqamous metaplasia (AIM) was initially introduced in 1983 to describe lesions in uterine cervix featuring a uniform intraepithelial full thickness basal cell proliferation with high nuclear density in absence of maturation but without sufficient criteria for diagnosis of high grade cervical intraepithelial neoplasia (CIN 3). [11]AIM has poor 


\section{Research Article}

intra and interobserver reproducibility on routine $\mathrm{H} \& \mathrm{E}$ stained sections because of its resemblance to CIN $3[12]$.

P16INK4A, a sensitive marker of cells with active expression of E7 oncoprotein, has also shown high sensitivity and specificity to HSIL in adult women [13]. Interaction of high risk HPV E7 gene product with $\mathrm{pRb}$, results in the liberation of $\mathrm{E} 2 \mathrm{~F}$, inactivation of $\mathrm{pRb}$ and stimulation of the S-phase of the cell cycle. This is strongly associated with p16INK4A expression. P16 tends not to be expressed in either normal proliferative epithelium cells or inflammatory lesions [13.14].

A marker of proliferation Ki67 has been shown to be a sensitive and specific marker of HPV infection in mature squamous epithelia [15]. Ki67 usually expressed in the second or third parabasal layers and rarely in the basal layer of the cervical squamous epithelium.

Cytokeratin (CK) 17 is a marker for endocervical reserve stem cells which give rise to metaplasia and expression of CK17 decreases and disappears as the metaplastic epithelium matures. Antibody to CK17 is used to differentiate between immature sqamous metaplasia (ISM) and high grade CIN (CIN3) [16].

The aim of study is to analyse the staining patterns of p16, Ki67 and CK17 in benign cervical lesions, cervical intraepithelial neoplasia and atypical immature squamous metaplasia and to evaluate their utility in differentiating cervical intraepithelial neoplasia from benign lesions and atypical immature squamous metaplasia of uterine cervix.

\section{Materials and Methods}

This cross-sectional study was conducted in the Department of Pathology, Baba Raghav Das Medical
College, Gorakhpur, UP on a total of 75 cases of formalin- fixed paraffin embedded cervical specimens of various cervical lesions comprising of benign lesions, cervical intraepithelial neoplasia (CIN) and atypical immature metaplasia(AIM) from August 2015 to October 2016. Inclusion criteria were all the female patients of age ranging from 21-70years presenting with various cervical lesions and who agreed to sign on consent form. Inadequate and autolysed tissue sample and the patients who did not adhere to the guidelines of protocols were excluded.

Histological sections were stained with Haematoxylin and Eosin stain for morphological diagnosis after concordance of a double blind evaluation by two independent pathologists. The cases were classified as benign lesions, CIN and AIM. Cases with either dissimilar diagnosis or with unsatisfactory material for evaluation were excluded from the study. Immunohistochemical staining for Ki-67, p16 and CK17 antigens was performed on all the cases using avidin-biotin peroxidase complex method and their immunoexpression were evaluated.

The performance of the immunohistochemical tests for p16, Ki67 and CK 17, in the detection of above mentioned cervical lesions was statistically evaluated by means of conventional contingency tables to calculate sensitivity, specificity, positive and negative predictive values considering the histological diagnosis as gold standard.The data was analysed statistically by applying $\mathrm{Z}$ test in SPSS version 23 . $\mathrm{P}$ value $<0.05$ was considered statistically significant.

The present work has been conducted after getting ethical clearance from the institutional ethical committee.

\section{Results}

The patients ranged from 21-70 years with a mean of $41.26 \pm 9.76$. Out of total 75 cases, 24 cases (32\%) were of benign lesions, 28 cases $(37.33 \%)$ of atypical immature metaplasia and 23 cases (30.66\%) were CIN. Among 23 cases of CIN, 4 cases were LSIL and 19 cases were HSIL.

Table-1: Comparative evaluation of p16, Ki67 and CK17 expression in benign, CIN and AIM lesions of cervix

\begin{tabular}{|l|l|l|l|}
\hline IHC Markers & $\begin{array}{l}\text { Benign }(\mathbf{n}=\mathbf{2 4} \text { cases }) \\
{[\text { No. }(\%)]}\end{array}$ & $\begin{array}{l}\text { CIN }(\mathbf{n = 2 3} \text { cases }) \\
{[\text { No. } \mathbf{( \% )})}\end{array}$ & $\begin{array}{l}\text { AIM }(\mathbf{n}=28 \text { cases }) \\
{[\text { No. }(\boldsymbol{\%})]}\end{array}$ \\
\hline p16 & $00(00.00 \%)$ & $21(91.30 \%)$ & $08(28.57 \%)$ \\
\hline Ki67 & $00(00.00 \%)$ & $18(78.26 \%)$ & $08(28.57 \%)$ \\
\hline CK17 & $03(12.50 \%)$ & $13(46.42 \%)$ & $28(100 \%)$ \\
\hline
\end{tabular}




\section{Research Article}

Table-2: Comparative evaluation of p16, Ki 67 and CK17 in diagnosing AIM versus CIN

\begin{tabular}{|l|l|l|l|l|}
\hline IHC Markers & AIM(N=28) & CIN(n=23) & Z Score & P value \\
\hline p16 & 8 & 21 & 4.5010 & $<0.001$ \\
Ki 67 & 8 & 18 & 3.5321 & $<0.001$ \\
CK 17 & 28 & 13 & 3.8942 & $<0.001$ \\
\hline
\end{tabular}

Table-3: Sensitivity and Specificity of p16, Ki67 and CK17 in detection of CIN

\begin{tabular}{|l|l|l|l|l|}
\hline IHC markers & Senstivity & Specificity & PPV & NPV \\
\hline p16 & $91.3 \%$ & $84.61 \%$ & $72.41 \%$ & $95.65 \%$ \\
Ki67 & $78.26 \%$ & $84.61 \%$ & $69.23 \%$ & $89.79 \%$ \\
CK17 & & & $32.5 \%$ & $67.74 \%$ \\
\hline
\end{tabular}

On analyzing the expression of p16, Ki 67 and CK 17 markers, out of 24 cases of benign lesions, none showed expression of p16 and Ki 67 while 3 cases $(12.5 \%)$ were positive for CK17.

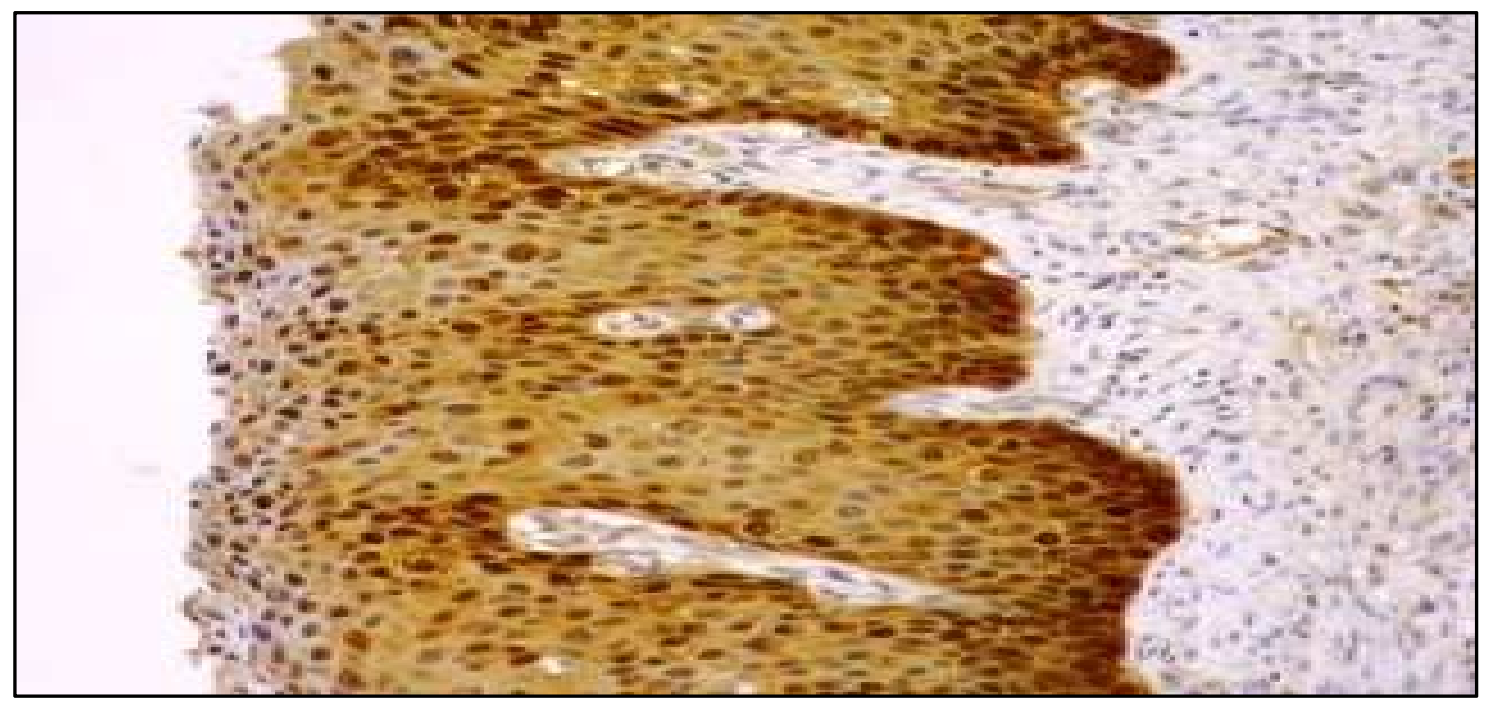

Fig.-1: Microphotograph of CIN 3 showing diffuse staining with p16

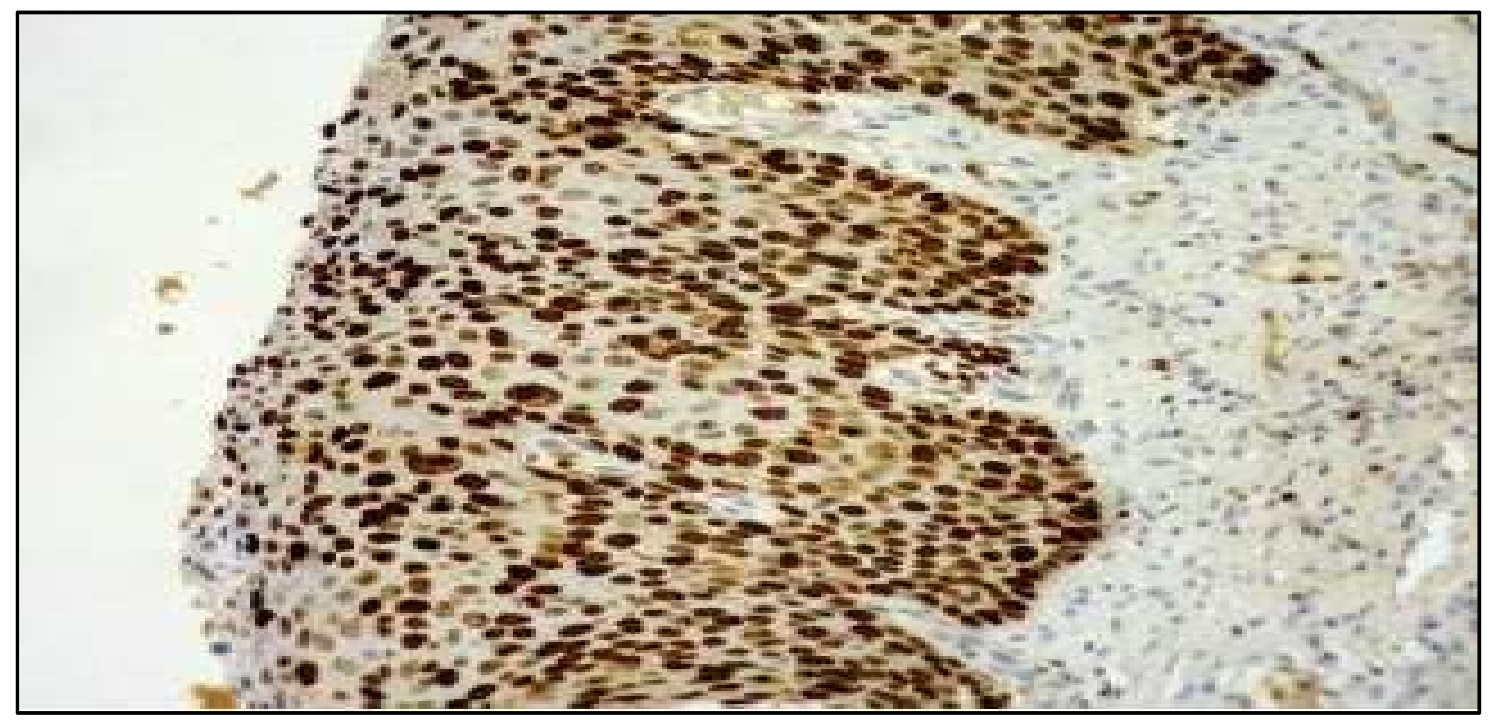

Fig.-2: Microphotograph of CIN 3 showing strong positivity with p16 
Among 23 cases of CIN, all the cases of HSIL (19 cases, 100\%) showed strong diffuse positivity while 2 out 4 cases of LSIL showed positive expression of p16. Ki 67 positivity was observed in 17 cases (89.47\%) of HSIL and one case $(25 \%)$ of LSIL respectively while CK17 expression was seen in 10 out of 19 cases (52.63\%) of HSIL and 3 of 4 cases(75\%) of LSIL.(Fig.1,Fig.2)

Among 28 cases of AIM, CK17 expression was observed in all cases while 8 cases $(28.57 \%)$ expressed both p16 and Ki67. (Table 1) $\mathrm{P}$ value of all the three markers in differentiating AIM from CIN is $<0.001$, which is found to be highly significant. (Table 2)

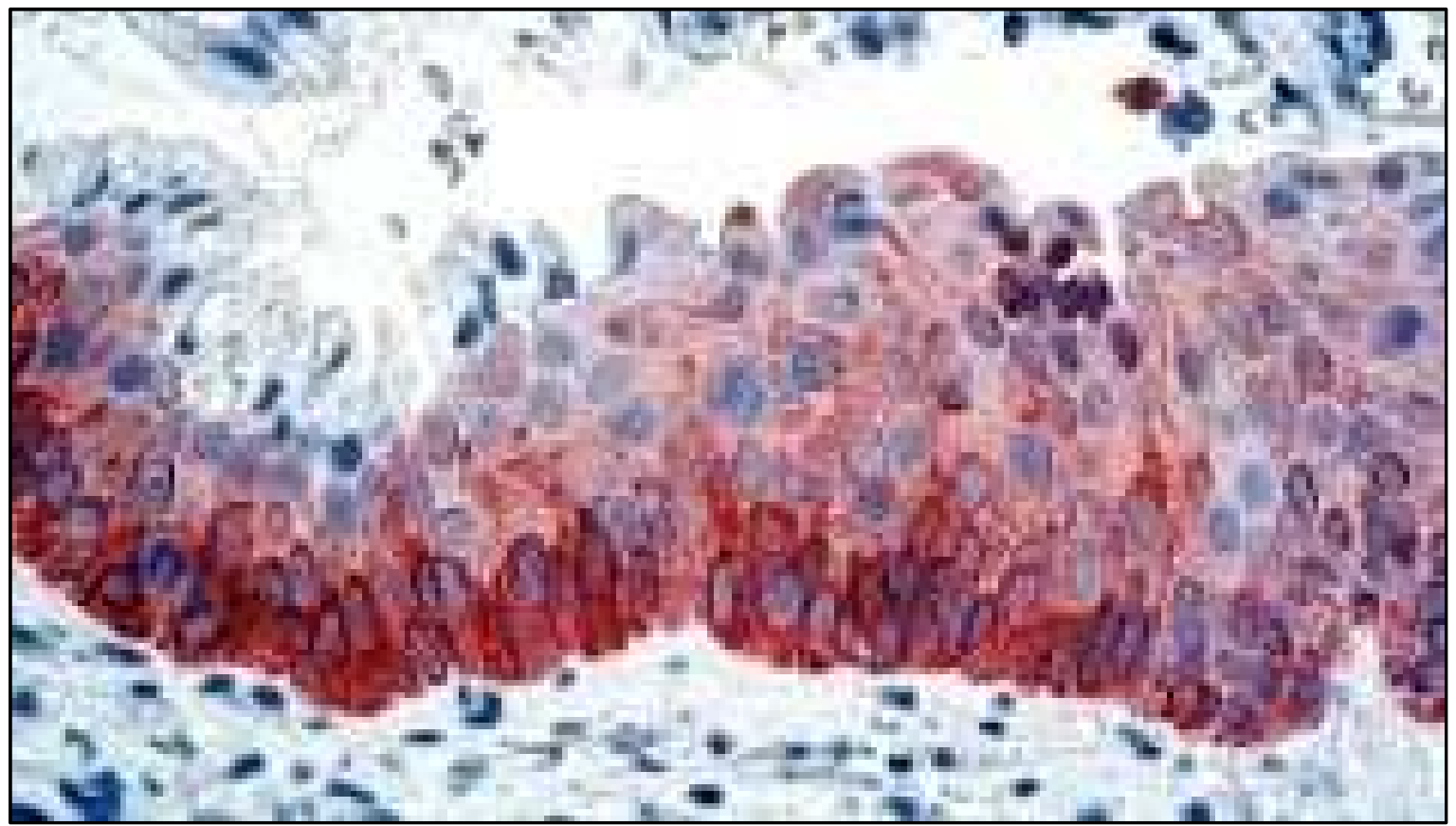

Fig.-3: Microphotograph of Atypical immature metaplasia showing positivity with CK17

The differences of expression of p16, Ki67 and CK17 between benign lesions versus CIN was <0.001, which was also found to be highly significant.

The sensitivity and specificity of p16 staining in detection of CIN were $91.3 \%$ and $84.61 \%$ respectively with positive predictive value (PPV) of $72.41 \%$ and negative predictive value (NPV) of $95.65 \%$.

The sensitivity and specificity of Ki67 were $78.26 \%$ and $84.61 \%$ with $69.23 \%$ PPV and $89.79 \%$ NPV respectively while the sensitivity and specificity of CK17 in detection of CIN were $56.52 \%$ and $40.38 \%$ respectively but its sensitivity in detection of atypical immature squamous metaplasia(AIM) were $100 \%$ in our study. (Table 3)

\section{Discussion}

Almost all of the invasive cervical cancers are preceded by cervical intraepithelial neoplasia (CIN).High rates of HPV infection is seen in adolescents and young women, persistence of which lead to development of premalignant lesions (CIN) and cervical cancer. Discordance on histological diagnosis of cervical cancer precursor lesions have been documented in several studies, suggesting a need to identify biological markers that could help the pathologist to make a correct diagnosis in equivocal lesions $[9,17,18]$. In adolescent and young adult women atypical immature metaplasia of the cervix, an immature metaplastic epithelium with mild cytological atypia but with strong reaction phenomenon, sometimes, mimics the morphology of high grade lesions. Therefore HSIL diagnosed lesions in this age group could in reality be a false positive. Testing for p16 expression appears to be a good addition to more accurately diagnose HSIL [10]. 


\section{Research Article}

In the present study, p16 was not expressed in any of the benign lesions, while all cases of HSIL (19 out of 19 cases, $100 \%$ ) showed strong positivity for p16. The staining was both nuclear and cytoplasmic and mostly involved full thickness of epithelium. Also p16 was positive in 2 of 4 LSIL cases $(50 \%)$ of which one was diffuse basal and the other diffuse one third thickness. Out of 28 cases of AIM, 8 cases $(28.57 \%$ ) showed positive expression of $\mathrm{p} 16$, while $71.4 \%$ were negative for $\mathrm{p} 16$.

Reuschenbach et al found the similar results [7]. The results of present study are also in agreement with the study conducted by Benevolo et al and Focchi et al [20, 21]. In their study, they also reported $100 \%$ positivity in cases of HSIL while none of the benign lesions expressed $\mathrm{p} 16$.

The sensitivity and specificity of p16 staining were $91.3 \%$ and $84.61 \%$ respectively with $72.41 \%$ PPV and $95.65 \%$ NPV. Present study findings match with the study of Walts et al in which sensitivity and specificity of p16 in detection of CIN was $89 \%$ and $83 \%$ respectively. PPV was $86 \%$ while NPV was $94 \%$ while Aslani FS reported $91.30 \%$ sensitivity, $98.10 \%$ specificity with $95.40 \%$ positive predictive value (PPV) and96.30\% negative predictive value (NPV) [22, 23].

Ki-67 is a cell proliferation marker demonstrated in some studies to aid in the diagnosis of HSIL. In the present study, Ki 67 expression was completely absent in benign lesions while 8 out of $28(28.57 \%)$ cases of AIM showed positivity for Ki67. Among CIN cases, 17 $(89.47 \%)$ of HSIL were positive for Ki67, while it showed positivity in 1 of $4(25 \%)$ LSIL cases. The present study showed statistically significant positive relation between proliferative activity, distribution of Ki67 positive cells and increasing CIN grade.

Our findings were in close agreement with the observation of Aslani FS et al study [23]. In their study they found Ki67 expression in $100 \%$ cases of HSIL, 25\% cases of LSIL, while none of benign lesions were positive. Kruse et al have found that there was a significant relation between CIN grade and number of Ki67 positive cells by using QPRODIT lineage analyzing system [24]. The distribution of Ki67 positive cells was related with CIN grade but there was overlap between CIN2 and CIN3 lesions similar to our study. Al-Saleh et al also found higher densities of Ki67 positive cells in HSIL than LSIL lesions [25].
The sensitivity and specificity of Ki67 staining were $78.26 \%$ and $84.61 \%$ respectively with $69.23 \%$ PPV and $89.79 \%$ NPV. This was in agreement with the Walts et al study [22].

On analyzing the expression of CK17 in AIM, all cases of AIM (28 cases, 100\%) were positive. 3 out $24(12.5 \%)$ cases of benign lesions expressed CK17, while among CIN , 3 out of 4 cases $(75 \%)$ of LSIL and 10 out of 19 cases $(52.63 \%)$ of HSIL showed positivity. These findings are well in accordance with the study of Aslani FS et al [23].

The sensitivity and specificity of CK17 in detection of CIN came out to be $56.52 \%$ and $40.38 \%$ respectively which are comparatively lower than p16 and Ki67 but its sensitivity in detection of AIM come out to be $100 \%$.CK 17 is a marker for basal cell of complex epithelia whose expression doesn't correlate with HPV infection or dysplasia [18]. In our view, CK17 expression in pseudostratified epithelia merely reflects a metaplastic phenotype/process. The dual expression of CK17 and p16 in atypical squamous lesions with metaplastic features rather supports the hypothesis of Ma et al that CIN III alternatively may develop via HPV infection of metaplastic cell [26].

\section{Conclusion}

p16, CK17 and Ki67 immunomarkers had a high degree of sensitivity and specificity and can be used as reliable diagnostic adjuncts to improve the routine diagnosis and reduce interobserver variability in cervical biopsy specimens. Immunohistochemical markers such as p16 alone or with Ki67 proved to be an important tool for the pathologists in distinguishing high grade cervical dysplasia from its benign mimics such as reactive inflammatory lesion and AIM thus avoiding overtreatment. However, complementary study including more cases and follow up examinations is warranted for better evaluation and definitive prognostic significance of these markers.

Funding: Nil, Conflict of interest: None initiated, Permission from IRB: Yes

\section{References}

1. Denny L. Cervical cancer: prevention and treatment. Discov Med. 2012Aug; 14(75):125-131. 
2. Fahey MT, Irwig L, Macaskill P. Meta-analysis of Pap test accuracy. Am J Epidemiol.1995Apr 1; $141(7 s): 680-9$

3. Sherman ME, Schiffman MH, Lorinez AT, Manos MM, Scott DR, Kuman RJ, et al. Toward objective Quality assurance in cervical cytopathology: Correlation of cytopathologic diagnosis with detection of high risk human papillomavirus types. Am J Clin Pathol.1994Aug; 102(2):182-7

4. Singh N. HPV and Cervical cancer - prospectus for prevention through vaccination. Ind $\mathrm{J}$ Med Paedia Oncol.2005; 26(1):20-3

5. Rosai J. Female reproductive system. In: Houston M, editor. Rosai and Ackerman's surgical pathology. 9th Ed. New York: Mosby; 2004. pp. 1523-68.

6. Nam EJ, Kim JW, Hong JW, Jang HS, Lee SY, Jang $\mathrm{SY}$, et al. Expression of the p16 and Ki-67 in relation to the grade of cervical intraepithelial neoplasia and highrisk human papillomavirus infection. J Gynecol Oncol. 2008Sep; 19(3):162-8.

7. Reuschenbach M, Seiz M, von KnebelDoeberitzC, Vinokurova S, Duwe A, Ridder R, et al. Evaluation of cervical cone biopsies for coexpression of p16INK4a and Ki-67 in epithelial cells. Int J Cancer. 2012Jan 15;130(2):388-94

8. Keating JT, Cviko A, Riethdorf S, Riethdorf L, Quade BJ, Sun D, et al. Ki-67, cyclin E, and p16INK4 are complimentary surrogate biomarkers for human papilloma virus-related cervical neoplasia. Am J Surg Pathol. 2001Jul; 25(7):884-91

9. Klaes R, Benner A, Friedrich T, Ridder R, Herrington S, Jenkins D, et al. p16INK4a immunohistochemistry improves interobserver agreement in the diagnosis of cervical intraepithelial neoplasia. Am J Surg Pathol. 2002Nov; 26(11):138999

10. Galgano MT, Castle PE, Atkins KA, Brix WK, Nassau SR, Stoler MH. Using biomarkers as objective standards in the diagnosis of cervical biopsies. Am J Surg Pathol. 2010Aug; 34(8):1077-87.
11. Crum CP,Egawa K, Fu YS et al. Atypical immature metaplasia(AIM). A subset of human papillomavirus infection of the cervix. Cancer 1983;51:2214-2219

12. Wentzensen $\mathrm{N}$, von Knebel Doeberitz $\mathrm{M}$. Biomarkers in cervical cancer screening. Dis Markers.2007; 23:315-30

13. Eleutério J Jr, Giraldo PC, Gonçalves AK, Cavalcante DI, de Almeida Ferreira FV, Mesquita SM, et al. Prognostic markers of high-grade squamous intraepithelial lesions: The role of p16INK4a and highrisk human papillomavirus. Acta Obstet Gynecol Scand 2007; 86(1):94-8

14. Cavalcante DM, Linhares LM, Pompeu MML, Givaldo PC, Eleutério J.The utility of p16 ${ }^{\mathrm{INK} 4 \mathrm{a}}$ and Ki67 to identify high-grade squamous intraepithelial lesion in adolescents and young women.Ind $\mathbf{J}$ Pathol Microbiol 2012;vol55(3):339-342

15. Iaconis L, Hyjek E, Ellenson LH, Pirog E. p16 and Ki-67 immunostaining in atypical immature squamous metaplasia of the uterine cervix. Correlation with Humam papillomavirus detection. Arch Pathol Lab Med 2007Sep;131(9):1343-49

16. Malpica A, Robboy SJ. Cervical benign and non neoplastic conditions. In: Robboy Js, Mutter GL, Prat J, Bentley RC, Russel P, Macolm C. Robboy's pathology of the female Reproductive Tract. $2^{\text {nd }}$ ed. London: Churchill livingstone; 2009.p.141-72

17. Kalof AN, Evans FM, Simmons-Arnold L, Beatty BG, Cooper K. p16 ink4a immunoexpression and HPV in situ hybridization signal patterns:potential markers of high grade cervical intraepithelial neoplasia. AM J Surg Pathol. 2005May; 29(5):674-9

18. Agoff SN, Lin P,Morihara J,Mao C, Kiviat N, Koustky L.p16ink4aexpression correlates with the degree of neoplasia: A comparison with ki67 expression and detection of high risk types. Mod Pathol. 2003Jul; 16(7):665-73

19. Benevolo M, Mottolese M, Marandino F, Vocaturo G, Sindico R, Piperno G et al. iimmunohistochemical expression of p16 ink4a is predictive of HR-HPV infection in cervical low grade lesions. Mod Pathol. 2006 Mar; 19(3):384-91 


\section{Research Article}

20. Focchi GR, Silva ID, Nogueira-de-Souza NC, Dobo C, Oshima CT,Stavale JN. Immunohistochemical exprerssion of p16ink4a in normal uterine cervix, nonneoplastic epithelial lesions and low grade squamous intraepithelial lesions. J Low Genit Tract Dis .2007Apr; 11(2):98-104

22. Walts AE, Bose S. P16/ki67 immunostaining is useful in stratification of atypical epithelium of the cervix. Clin Med Pathol 2008Mar; 1:35-42

23. Aslani FS, Safaei A, Pourjabali M , Momtahan M.Evaluation of ki67,p16 and ck17 markers in differentiating cervical intraepithelial neoplasia and benign lesions. Iran J Med Sci.2013Mar;38(1): 15-21
24. Kruse AJ, Baak JP, de Bruin PC, Jiwa M, Snijders WP, Boodt PJ. Ki 67 immunoquantitation in cervical intraepithelial neoplasia (CIN): A sensitive marker for grading. J Pathol.2001 Jan; 193(1): 48-54

25. Al-Saleh W, Delvenne P, Greimers R, Fridman V, Doyen J, Boniver J. Assesment of ki67 antigen immunostaining squamous intraepithelial lesions of the uterine cervix. Correlation with the histologic grade and human papillomavirus type.Am J Clin Pathol 1995Aug;104(2):154-60

26. Ma L, Fisk JM, Zhang RR, Ulukus EC, Crum $\mathrm{CP}$. Zheng W. Eosinophilic dysplasia of the cervix: a newly recognized variant of cervical squamous intraepithelial neoplasia. Am J Surg Pathol. 2004Nov; 28(11):1474-84.

\section{How to cite this article?}

Mitra S.K., Misra R.K., Varshney B., Kumari A., Rai D. Role of p16, Ki67 and CK17 in differentiating benign lesions, cervical intraepithelial neoplasia(CIN) and atypical immature squamous metaplasia(AIM) of uterine cervix. Trop J Path Micro 2017;3(1):13-19.doi: 10.17511/jopm.2017.i1.03. 